\title{
Computed tomography-guided percutaneous biopsy for vertebral osteomyelitis: a department's experience
}

\author{
Vasant Garg, M.D., Christos Kosmas, M.D., Peter C. Young, M.D., \\ Uday Kiran Togaru, M.B.B.S., ANd Mark R. Robbin, M.D. \\ Department of Radiology, University Hospital Case Medical Center, Cleveland, Ohio
}

\begin{abstract}
Object. Vertebral osteomyelitis has been reported to occur in approximately $0.2-2$ cases per 100,000 annually. Elevated laboratory values such as erythrocyte sedimentation rate and C-reactive protein suggest inflammatory etiologies. Different imaging modalities, from radiography and CT scanning to nuclear medicine imaging and contrastenhanced MRI, can be employed to evaluate for osteomyelitis. Although MRI has a strong sensitivity and specificity for vertebral osteomyelitis, obtaining histological and microbiological samples remains the gold standard in diagnosis. Therapy can be geared toward the specific pathogen cultured, thereby preventing the need surgical intervention in the majority of cases. However, recent reports have questioned the percentage yield of image-guided percutaneous biopsy even when there is a high clinical suspicion for vertebral osteomyelitis.

Methods. After obtaining institutional review board approval, the authors performed a chart review of patients who had undergone image-guided percutaneous bone biopsies at University Hospitals Case Medical Center in Cleveland, Ohio. Data were filtered for patients in whom a biopsy sample of a vertebral body/disc was obtained. A total of 213 procedures were performed, of which clinicians indicated a concern for infection in 84, infection or neoplasm in 13 , and a noninfectious etiology (the majority being neoplasms) in the remaining 116.

Results. Histological examination provided positive results in $25(41.0 \%)$ of the 61 samples collected for suspected cases of osteomyelitis. Microbiology samples were less predictive, with only 16 of the 84 samples collected, or $19.0 \%$, yielding a positive result. In 10 patients there were positive blood and/or urine cultures. Of these, 8 samples $(80 \%)$ demonstrated the same pathogen identified by biopsy (for the remaining 2 positive systemic cultures, no pathogen was identified by the percutaneous intervention). In other words, half of the 16 cases that provided microbiological results from biopsy demonstrated the same results by systemic cultures. However, $89(76.7 \%)$ of the 116 samples collected with the primary concern of neoplasm yielded results.

Conclusions. Image-guided percutaneous biopsy for vertebral osteomyelitis demonstrates an extremely low probability of identifying specific microbes. Blood or urine cultures concurrently identified culprit pathogens in $50 \%$ of positive biopsy cultures. Therefore, in only $8(9.5 \%)$ of 84 biopsies did the biopsy results provide additional information to clinicians as to the pathological microorganism present and how treatment might need to be adjusted. (http://thejns.org/doi/abs/10.3171/2014.6.FOCUS14134)
\end{abstract}

\section{KEY WORDS • CT-guided biopsy $\quad$ - vertebral osteomyelitis $\quad \bullet \quad$ spondylitis}

$\mathrm{I}$ NFECTIONS have been and will always serve as one of the main sources of pathologies. Although the vast majority involve the respiratory, gastrointestinal, and genitourinary organ systems, the vertebral bodies are not excluded from involvement and account for $2 \%-7 \%$ of musculoskeletal infections. ${ }^{5}$ Vertebral osteomyelitis is not an infrequent pathology and has been reported to occur at approximately $0.2-2$ cases per 100,000 annually. 5,6 Associated inflammatory septic discitis is extremely rare, seen in 1 of 250,000 and $2 \%-4 \%$ of all osteomyelitis infections. ${ }^{25}$

In the majority of cases, an insidious onset of back or neck pain is reported, often taking months to years to develop. ${ }^{5,6}$ Most back pain stems from degenerative pro-

\footnotetext{
Abbreviations used in this paper: $\mathrm{CRP}=\mathrm{C}$-reactive protein; $\mathrm{ESR}$ $=$ erythrocyte sedimentation rate; $\mathrm{WBC}=$ white blood cell.
}

cesses; however, a population-based study by Carey et al. indicated that up to $7.6 \%$ of acute back pain and $3.9 \%$ of chronic pain stems from vertebral osteomyelitis. ${ }^{3}$ A variety of associated symptoms exist including limited range of motion, discomfort, lethargy, confusion, anorexia, nausea and vomiting, and weight loss. Surprisingly, fewer than $20 \%$ of patients present with fever. Neurological symptoms are rare and are typically only seen when associated with mass effect-for example, in the presence of an abscess. ${ }^{5,6,22}$

A review by Tali found the prevalence of spinal infections to be twice as high in males as females, ${ }^{23}$ although more recent reports have not shown any major difference between sexes. Numerous risk factors are associated with spinal infections, the most common involving direct exposure of the vertebral bodies to pathogens introduced during prior surgery and trauma. Other risk 
factors include diabetes mellitus, malnutrition, substance abuse, HIV infection, malignancy, long-term steroid use, chronic renal failure, liver cirrhosis, decreased immune function, and septicemia. , $22^{2}$

Initial workup involves laboratory evaluation. White blood cell (WBC) count typically ranges from 4 to 23 thousand with an average at 11.8 thousand, just above the 10 - to 11-thousand upper limit of normal. Elevated erythrocyte sedimentation rate (ESR) and C-reactive protein (CRP) are nearly always present, with sensitivities reaching up to $90 \%$, albeit not specific for infection. ${ }^{5}$ The rule of $50 \%$ has been coined: $50 \%$ of patients are older than 50 years of age; symptoms last more than 3 months in $50 \%$; fever is present in $50 \%$; WBC count is normal in $50 \%$; genitourinary tract is the primary source in $50 \%$; Staphylococcus aureus is causative microbe in more than $50 \%$; lumbar spine involvement is present in 50\%; and no primary site is detected in $50 \% .^{23}$

Numerous imaging modalities may assist in the evaluation for vertebral osteomyelitis including radiography, nuclear medicine imaging, and MRI. Radiography may reveal late osseous destruction, but in an earlier stage of the disease, findings may resemble those of common degenerative processes, or findings may be completely absent. The utilization of radionuclide studies, including ${ }^{99 \mathrm{~m} T c}$ methylphosphonate bone scans, ${ }^{67} \mathrm{Ga}$ WBC scans, and ${ }^{111}$ In WBC scans, demonstrate increased avidity with osteomyelitis; however, like ESR and CRP laboratory values, high sensitivities exist but specificity is low and therefore wide differentials remain.5 Alternatively, the sensitivity of MRI for vertebral osteomyelitis is often reported between $76 \%$ and $100 \%$ (mean $91 \%$ ), with a corresponding specificity ranging from $65 \%$ to $96 \%$ (mean $82 \%) .{ }^{19}$ Furthermore, signal changes identified by MRI can be seen in early stages of the infection, and therefore MRI serves as the gold standard in imaging modalities.

Nevertheless, practice currently recommends the procurement of histological and microbiological samples in the confirmation and identification of specific organisms, thereby allowing appropriate treatment targeted to a specific microorganism. Obtaining a surgical sample will always be a viable option for confirming the diagnosis. Yet percutaneous biopsy with fluoroscopic or CT guidance has been proven as a viable, faster, and more cost-effective approach that is associated with fewer complications. ${ }^{17,24}$ The first percutaneous vertebral bone biopsy was described by Ball in 1934. ${ }^{1}$ The utilization of image guidance was first seen with radiography in 1949, fluoroscopy in 1969, CT in 1981, MRI in 1986, and CT fluoroscopy in 1996. ${ }^{24}$

A review of the literature has suggested a wide range in the success rates for percutaneous biopsy. Many studies from the 1990s and early 2000s reported the accuracy of a percutaneous biopsy of vertebral lesions with CT or fluoroscopy guidance ranging from $88 \%$ to $100 \% .^{24} \mathrm{~A}$ recent retrospective review by Sehn and Gilula of 113 cases in which percutaneous biopsy was used for the diagnosis and identification of causative organisms poses a contrast to the results from the 1990 s and 2000 s, with a positive histological result found in $63(55.7 \%)$ of 113 cases and a positive microbiological result found in only 28 (30.4\%) of 92 cases. ${ }^{22}$ The musculoskeletal radiologists at University Hospitals Case Medical Center shared a similar suspicion for the low yield of image-guided percutaneous biopsy in cases of vertebral osteomyelitis.

\section{Methods}

A retrospective case review was performed of all patients who underwent a CT-guided percutaneous bone biopsy for vertebral osteomyelitis over an approximately 9-year period, specifically between January 2005 and October 2013. All procedures were performed at University Hospitals Case Medical Center in Cleveland, Ohio, which serves as a Level II academic center. Initial approval for the retrospective review was obtained from the institutional review board. Each intervention had already been performed, so no patients were placed at any additional risk. Furthermore, no financial incentive was given to any of the patients. Upon receiving institutional review board approval, a retrospective review of the institution's radiology PACS system was performed by searching the procedure code used to bill for percutaneous bone biopsy, which yielded over 600 results. The list was filtered to include only those patients who had received a vertebral bone biopsy summing a total of 213 procedures on 209 patients.

These 213 vertebral bone biopsies were categorized according to the ordering clinician's level of suspicion for osteomyelitis and the indication listed on the radiology intervention requisition. The study excluded a subset of 116 patients, the majority of whom had known malignancy, and the primary purpose of the study was to investigate for the presence of metastases. A smaller group of 13 cases was used to investigate for any etiology, with the range in order of decreasing level of suspicion including infection or neoplasm, degenerative changes, and inflammatory processes. This study focuses on a cohort of 84 patients. Of these, 4 patients had procedures performed on 2 separate occasions: 2 of the procedures were for a suspected neoplasm and 2 were for suspected osteomyelitis. For the 2 patients who had procedures performed twice for osteomyelitis, multiple months elapsed before a recurrence of symptoms warranting the second biopsy.

The primary clinician first indicated whether there was a strong clinical suspicion for vertebral osteomyelitis, taking into consideration advanced imaging findings in correlation with the patient's history and laboratory values. However, the musculoskeletal radiologist made the final decision regarding whether the entire picture fit that of vertebral osteomyelitis and whether to perform the percutaneous biopsy.

Our institution does not have a standardized method to include or exclude patients from having the procedure. However, the musculoskeletal radiologists required that all patients have some form of advanced imaging suggesting an infectious process prior to the procedure. Of the 84 patients who underwent the procedure, $80(95.2 \%)$ underwent immediate pre-biopsy advanced imaging. Of these 80 patients, $78(97.5 \%)$ had pre-biopsy MRI studies that raised concern for osteomyelitis/discitis. In the vast majority of these MRI-suspicious cases, the radiologists 
were confident in the diagnosis of osteomyelitis/discitis and/or periligamentous fluid collection concerning for infection without proposing a differential diagnosis. This study did not focus on statistically comparing the MRI reports. For the remaining 2 of 80 patients who underwent advanced imaging other than MRI, CT was performed and followed by nuclear medicine imaging including PET in 1 case, and bone scanning and WBC scanning in the second case. In both patients, the pre-biopsy nuclear imaging raised concern for an inflammatory or infectious process (Figs. 1-3).

For the 4 patients without imaging prior to the biopsy, 2 were from satellite hospitals and information could not be collected during the chart review. One of the patients had numerous prior lumbar surgeries and complications; although information was not present to explain why prebiopsy advanced imaging was not performed, the screening CT performed on the day of the biopsy detected a periligamentous fluid collection, which was aspirated. For the last case without advancing imaging prior to the biopsy, the patient had known chronic osteomyelitis and is one of the two patients mentioned earlier in the paper who had a percutaneous biopsy for vertebral osteomyelitis performed on 2 separate hospital visits. For this patient, MRI had been performed prior to the first biopsy, and the second biopsy was performed in the same location as seen on this MR image. Therefore, when taking these limitations into consideration, including incomplete charting in the electronic medical records, and also taking into consideration the patient's history and advanced imaging, $84(100 \%)$ of the 84 patients had advanced imaging prior to the biopsy.

Biopsy was performed in all cases under CT guidance. Complete blood count, prothrombin time, and inter-
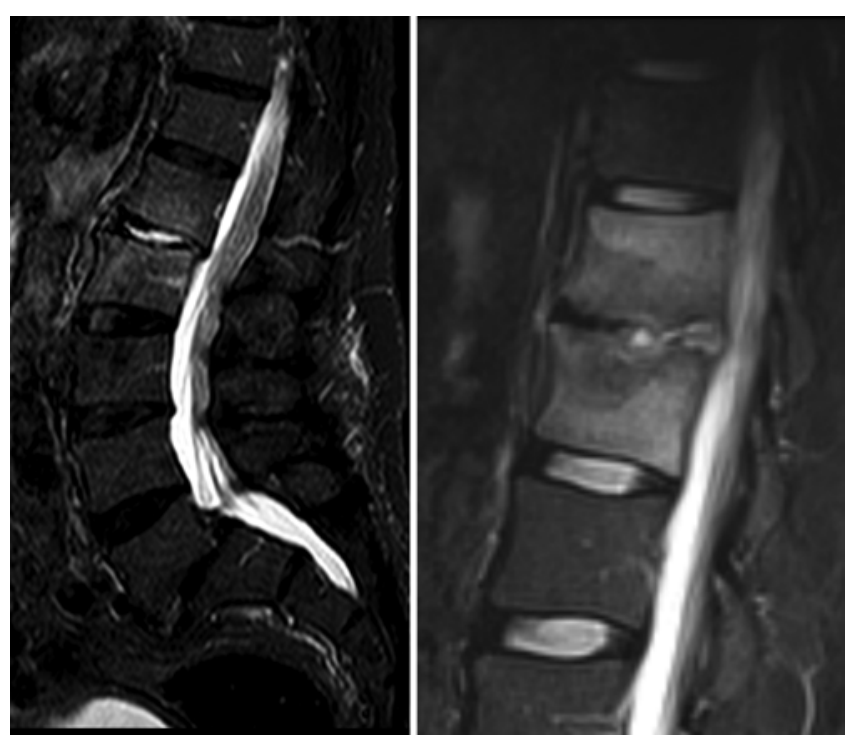

FIG. 1. Left: Sagittal STIR image demonstrating increased signal in the intervertebral disc space, corresponding to fluid, with mild marrow edema in the vertebral bodies. No endplate erosion is seen. Right: Sagittal STIR image from another patient again demonstrating increased signal in the intervertebral disc space corresponding to fluid; in addition, there is cortical irregularity of the endplates and marked marrow edema in the vertebral bodies. national normalized ratio were obtained prior to the procedure. A preprocedural 1- to 2-mm thin-slice CT scan covering the area of interest was acquired, and, based on the results, the approach and site of skin puncture were determined. The biopsy was performed with the patient under conscious sedation or, in selected cases of comorbidities or inability of the patient to lie prone, under general anesthesia. Local anesthesia was also used, and the procedure was performed under sterile conditions.

Biopsy was performed using either a Laurane bone biopsy system (Laurane Medical) or a Bonopty bone biopsy system (Radi Medical Systems). The Laurane system consists of an 11-gauge external cannula with an internal stylet and a 12.5-gauge trephine biopsy needle. The Bonopty system consists of 14-gauge external cannula and internal drill and a 16-gauge trephine biopsy needle. In both systems the external cannula and stylet/drill were inserted into the bone and then the internal stylet/drill was replaced by the trephine biopsy cutting needle, and the biopsy was performed. In the lumbar spine the approach was transpedicular, whereas in the thoracic spine it was transpedicular or intercostovertebral. The patients' vital signs were monitored throughout the procedure.

In general, our radiologist attempted to attain an aspiration of the disc and an adequate core sample from the vertebral endplate disc complex. When focal fluid collections were visualized, the procedure specifically targeted and aspirated these collections. If permitted, multiple core biopsy samples were collected. Often a satisfactory single core biopsy would be cut in half to be sent to both microbiology and pathology.

Our radiologists' primary goal was to gather a sufficient sample for microbiology and their secondary goal was to gather a sample for surgical pathology. Often when only a small or single sample was obtained, all of it was sent only to microbiology. The radiologist used clinical judgment during each individual procedure to determine whether a single or additional core biopsy should even be performed. For example, if it appeared that sufficient purulent fluid had been collected from a phlegmon or abscess, a core biopsy might have to be avoided to prevent further discomfort to the patient. In certain cases, patients could not tolerate further biopsy and this stopped the procedure.

\section{Results}

All samples were sent to the lab for bacterial Gram stain, culture, fungal culture, and mycobacterial culture. Samples were sent to pathology for histological evaluation in 61 of 84 cases.

At least 1 core sample was obtained in 74 of the 84 patients $(88.1 \%)$. Of the 10 patients in whom a core biopsy could not be performed, the procedural dictations specifically mentioned the aspiration of a sizeable fluid collection in 6 the patients and reportedly measuring up to 20 $\mathrm{ml}$. Three reports did not specify why a core biopsy was not collected, but in reiteration, the radiologist used clinical judgment on a case-by-case basis to determine how much sample was needed. One of the patients could not tolerate the procedure further, and therefore the procedure was aborted without a core sample being obtained. 

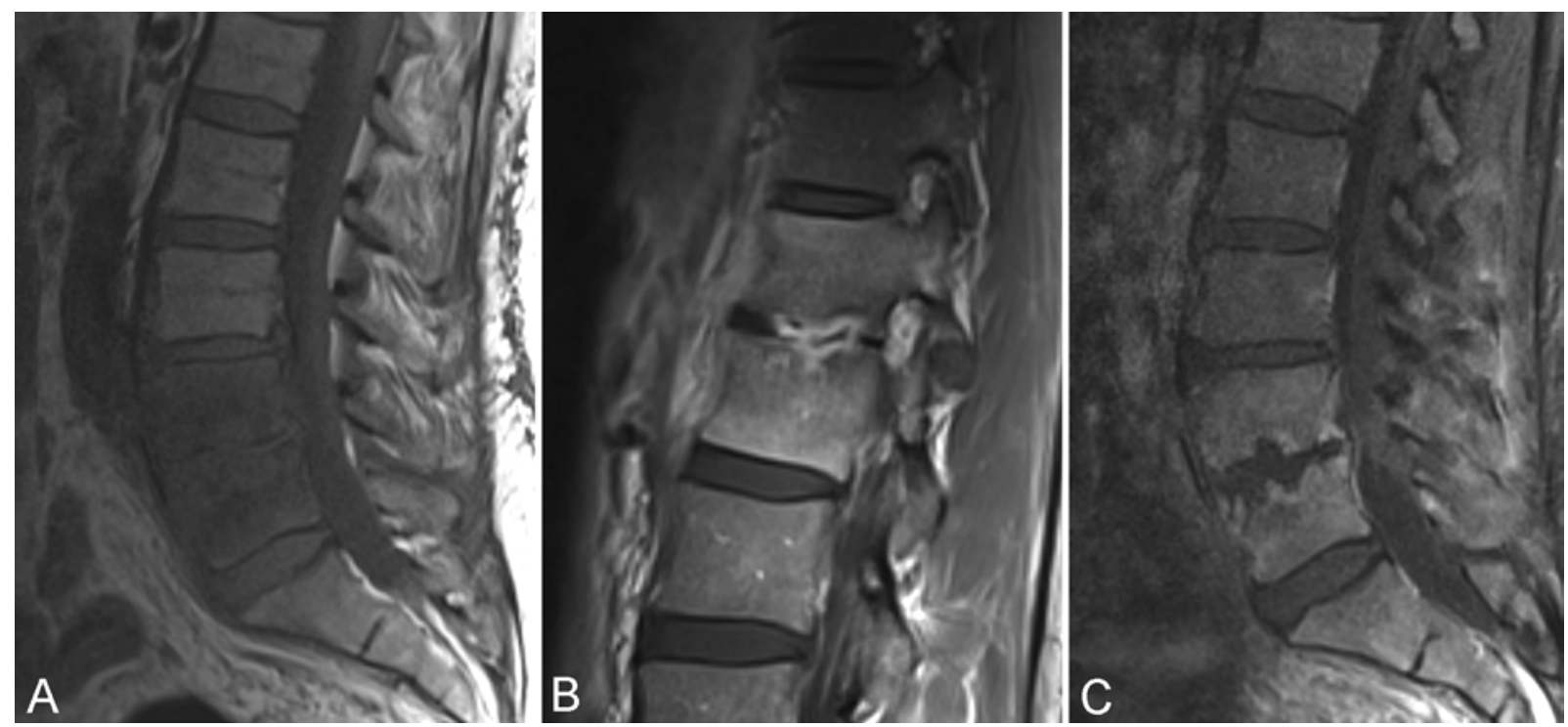

FIG. 2. A: Sagittal T1-weighted MR image demonstrating loss of normal marrow signal at the affected vertebral bodies with irregularity of the endplates. B: Sagittal fat-suppressed postcontrast T1-weighted MR image revealing enhancement in the disc space and in the vertebral bodies and lack of enhancement in the central aspect of the disc space corresponding to fluid; irregularity of the endplates is noted. C: Sagittal fat-suppressed postcontrast T1-weighted MR image from another patient again demonstrating enhancement of the vertebral bodies and lack of enhancement in the central aspect of the disc space corresponding to fluid, as well as more pronounced irregularity and erosions of the endplates.

On a few occasions, patients could not tolerate the initial aspect of the procedure and the patient had to be brought back at a different time, often requiring full sedation.

Histology was positive in 25 of the 61 samples collected, yielding a proportion of positive histology per total number of biopsies for osteomyelitis/discitis of $41.0 \%$. Microbiology was less predictive with only 16 of the 84 samples collected (19.0\%) yielding results. In 44 of the 84 total samples $(52.4 \%)$, the patients were not treated with antibiotics prior to the procedure. Of these 44 patients, 9 had positive samples, yielding a $20.5 \%(9 / 44)$ accuracy rate. Forty of the 84 patients $(47.6 \%)$ were treated with antibiotics prior to the procedure. Of these 40,7 had positive samples, yielding $17.5 \%$ (7/40) accuracy rate. Using chi-square analysis to compare the expected microbiology results of those patients not having been treated with antibiotics prior to the biopsy with the observed results from
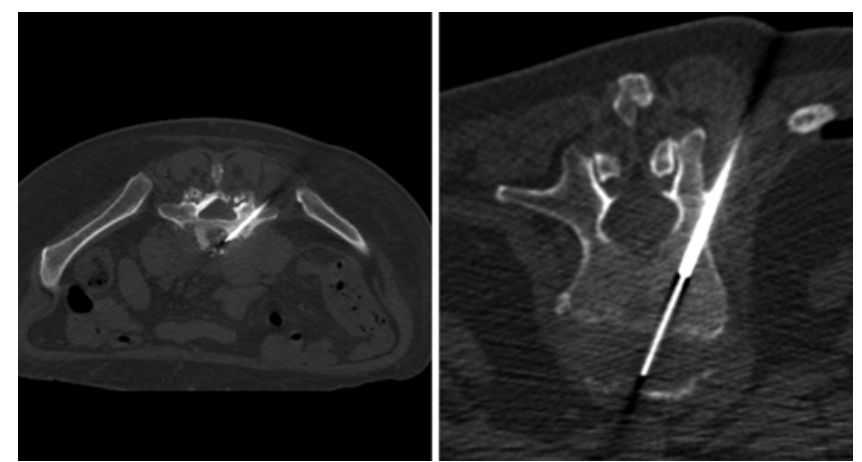

FIG. 3. Left: Axial CT scan demonstrating a bone biopsy needle inserted via a transpedicular approach. Right: Axial CT scan from another patient again demonstrating a bone biopsy inserted via a transpedicular approach. An 18-gauge Chiba needle has been advanced into the disc space for fluid aspiration. patients having been treated prophylactically, the 1-tailed $\mathrm{p}$ value was $0.4748(>0.05)$. Therefore, the difference in results between those not treated before biopsy and those treated prophylactically was not statistically significant.

Table 1 shows the yield of histology and microbiology based on the total number of cases collected when there was a clinical suspicion of vertebral osteomyelitis, and Table 2 shows the yield of microbiology according to the presence or absence of prophylactic antibiotic administration.

The majority of the biopsies were performed at the thoracic and lumbosacral levels, 37 and 46 cases, respectively, with only 1 cervical biopsy. Eleven of the 37 thoracic biopsies (29.7\%) were positive, whereas only 5 of the 46 within the lumbosacral region $(10.9 \%)$ and 0 of the 1 cervical case were positive. Eight of the 16 positive biopsy cultures (50\%) were of S. aureus. The remaining microorganisms are as follows: coagulase-negative Staphylococcus $(\mathrm{n}=3)$, Escherichia coli, Enterococcus faecium, Proteus mirabilis, Candida, and only Mycobacterium tuberculosis $(\mathrm{n}=1$ for each).

The yield of microbiology according to vertebral body level is shown in Table 3 .

Ten patients had positive blood and/or urine cultures.

TABLE 1: Yield of histology and microbiology per total number of cases collected when there was clinical suspicion of vertebral osteomyelitis

\begin{tabular}{lcc}
\hline & \multicolumn{2}{c}{ No. of Samples } \\
\cline { 2 - 3 } Results & Histology & Microbiology \\
\hline positive & 26 & 16 \\
negative & 42 & 68 \\
total & 68 & 84 \\
\hline
\end{tabular}


TABLE 2: Yield of microbiology in patient who received and patient who did not receive prophylactic antibiotic administration

\begin{tabular}{lccc}
\hline & \multicolumn{2}{c}{ Patient Received Antibiotics } & \\
\cline { 2 - 3 } Result & Yes & No & Change \\
\hline positive & 7 & 9 & 16 \\
negative & 33 & 35 & 68 \\
total & 40 & 44 & 84 \\
\hline
\end{tabular}

Of these, 8 of the 10 positive systemic cultures (80\%) demonstrated the same pathogen identified by percutaneous biopsy. The 2 cases of systemic infection without positive biopsy cultures were of $S$. aureus and coagulase-negative Staphylococcus. The high frequency of these pathogens suggests false negatives of the biopsy samples. Therefore, in 8 of 16 cases of positive microbiology established by percutaneous biopsy, the pathogens were also identified by positive blood or urine cultures. After factoring out the 8 cases in which the systemic culture provided the same results as the biopsy, only 8 , or half, of the 16 positive biopsy cultures provided additional information to the clinician concerning the pathological microbe present and how treatment might need to be adjusted. Only 8 of the 84 total biopsies with an initial high suspicion for osteomyelitis $(9.5 \%)$ provided additional information to the clinician concerning the pathological microbe present and how treatment might need to be adjusted. On the other hand, 89 of the 116 samples $(76.7 \%)$ collected with the primary concern of neoplasm yielded a diagnosis.

Limitations to this study must be acknowledged. Although each of the procedures was performed at the same institution by 3 board-certified musculoskeletal radiologists, varying intraprocedural circumstances prevented the acquisition of the exact same sample in each case. As mentioned earlier, each radiologist used clinical judgment during each individual procedure to determine whether a single or additional core biopsy should even be performed. For example, if it appeared that sufficient purulent fluid had been collected from a phlegmon or abscess, a core biopsy may have been avoided to prevent further distress to the patient. In certain cases patients could not tolerate further biopsy, stopping the procedure. As a result, only 68 pathology samples were collected from the 84 patients who had undergone the procedure. In addition, there was variability regarding sample type and size sent for microbiology analysis. Furthermore, the analysis of the data may have differed among the pathologists.

To collect the largest patient population for our study, the time frame of nearly 9 years meant that period dates

TABLE 3: Yield of microbiology stratified by vertebral body level

\begin{tabular}{lccc}
\hline \multicolumn{1}{c}{ Level } & Positive & Negative & Total \\
\hline cervical & 0 & 1 & 1 \\
thoracic & 11 & 26 & 37 \\
lumbosacral & 5 & 41 & 46 \\
total & 16 & 68 & 84 \\
\hline
\end{tabular}

back to 2005 when the institution first began utilizing PACS. Patients who had undergone an image-guided percutaneous vertebral biopsy may have been unaccounted for, although we considered this would represent only a small number of cases, if in fact any cases were actually missed. Furthermore, the institution transitioned from paper documents to electronic medical records in the early 2000s. It is not entirely impossible that data may have been lost during this transition.

To detail the follow-up and treatment of each patients is beyond the scope of this study. The study is based on the suspicion of vertebral osteomyelitis, and definitive osteomyelitis is not always known. A cursory review of discharge summaries for the 84 patients who underwent a vertebral biopsies for osteomyelitis indicated that 71 $(84.5 \%)$ were discharged on antibiotics. In the majority of these cases, the patients were on long-term antibiotics for osteomyelitis/discitis; however, it is beyond the scope of this study to perform a statistical analysis of which patients were treated specifically for vertebral osteomyelitis/discitis (or, alternatively, for example, sepsis), the length of treatment (and whether the length corresponded to what is recommended for the treatment of vertebral osteomyelitis/discitis), which type of antibiotics were used, whether the patients improved clinically, and so on.

Of the 13 patients for whom discharge paperwork did not specifically mention subsequent antibiotic treatment, 6 discharge summaries were not identified which is due to paperwork located at our satellite institutions or to limitations with electronic medical records as mentioned above. Only 7 discharge summaries (or follow-up outpatient notes) specifically mentioned the cessation of antibiotic use due to negative biopsy results, with only 1 case attributing the patient's back pain to metastasis (which did not correspond to our biopsy results). In effect, taking into consideration that there were only 7 post-biopsy reports specifically excluding an infectious process, 77 of 84 patients, or $91.7 \%$, continued to be treated with antibiotics after discharge.

\section{Discussion}

Although the correlation of clinical presentation and MRI imaging provides up to $100 \%$ sensitivity and specificity for vertebral osteomyelitis, ${ }^{19}$ biopsy remains the gold standard in diagnosis. Isolation of the causative organism is considered optimal to tailor appropriate antibiotic treatment. ${ }^{9}$ Mycobacterium tuberculosis is one of the most common infectious pathogens worldwide, albeit less prevalent in the West. It is also known to afflict the vertebral bodies, serving as the most common pathogen extracted from vertebral biopsy samples. ${ }^{5,7,15,17}$ It typically presents with a subacute clinical course. Magnetic resonance imaging demonstrates characteristic "skip lesions" in which contiguous vertebral bodies are affected without involvement of the intervertebral discs. The pathogen is notoriously difficult to culture, typically requiring 6-8 weeks for the isolation, ${ }^{7,21}$ although now it can be done faster using polymerase chain reaction.

In the United States, gram-positive cocci are the most common culprit microorganisms of discitis/osteomyelitis, specifically $S$. aureus and to a lesser degree Streptococ- 
cus. ${ }^{4,13,21}$ In a review of the literature, Cheung and Luk reported Staphylococcus and Streptococcus for more than $50 \%$ of vertebral osteomyelitis cases. ${ }^{5}$ Our results confirm this finding with 8 of 16 positive samples yielding Staphylococcus on culture. Escherichia coli is often seen with concurrent urinary tract infections, as seen in 1 patient in our study. Other correlations include gramnegative bacilli in intravenous drug abusers or coagulasenegative Staphylococcus and Streptococcus viridans in the immunocompromised. ${ }^{5}$ Even though much less common, physicians must take into account the possibility of fungal infections, which typically present as a result of Candidemia with the delayed complication of spondylodiscitis several months later. ${ }^{16}$ Rankine and colleagues stressed the importance of attaining fungal cultures in that Candida was isolated in 1 patient after treatment with flucloxacillin..$^{21}$ Our study also supports this finding, with 1 of 16 positive samples yielding Candida.

Endoscopic biopsies provide an approximately $90 \%$ yield for vertebral osteomyelitis. ${ }^{26}$ However, it is well known that percutaneous intervention is safer and provides quicker results at a lower cost and with decreased morbidity. ${ }^{17,24}$ As mentioned in the introduction, percutaneous vertebral biopsies were described as early as 1934 by Ball, and image guidance was subsequently added in 1949.,24 Computed tomography scanning is currently held to be the modality of choice for guidance by the majority of authors, although some authors, such as Kim et al. in 2013, have demonstrated equal success using fluoroscopy. ${ }^{17}$

Many retrospective studies and reviews have suggested a high success rate using percutaneous biopsy for vertebral lesions. In 2001, Chew and Kline ${ }^{6}$ performed a retrospective evaluation of spontaneous (non-postoperative) infectious discitis involving 105 biopsies in 92 patients, reporting a $97.1 \%$ success rate. In a 2007 review of percutaneous biopsy methods used in the spine, Tehranzadeh et al. referenced 8 articles with an accuracy rate approaching $88 \%-100 \% .{ }^{24}$ In a 2009 article entitled "CTguided fine-needle aspiration in vertebral osteomyelitis: true usefulness of a common practice," de Lucas et al. acknowledged 13 reports with accuracy results ranging from $71 \%-100 \%$. However, the paper clearly acknowledged that "these series usually mix many cases of neoplastic vertebrae and a minor portion of spinal infections. In addition, these series frequently described a relatively lower sensitivity in the subgroup of inflammatory cases rather than tumor cases." 10 It is already known that the role of image-guided biopsy in diagnosing malignant conditions is well established-89 of 116 samples (76.7\%) collected in our trial when there was an initial concern for neoplastic process were diagnostic - but its role in diagnosing infectious diseases is less accurate. ${ }^{11}$

It is therefore imperative to scrutinize how statistics are calculated and delineate findings according to neoplastic and nonneoplastic etiologies. Chew and Kline ${ }^{6}$ acknowledged that their high $91 \%$ diagnostic rate was based on the 39 samples with microorganisms identified out of 43 cases in which "active disc space infection" was present. "A patient without positive results from microbiology was considered not to have active disk space infection. The strict criteria for the presence of active disk space infection used for the purposes of this retrospective study are not necessarily the criteria that one might use in the clinical setting to guide patient care" (emphasis added). Following up their reference to the 8 articles with diagnostic results ranging between $88 \%$ and $100 \%$, Tehranzadeh and colleagues acknowledged that the ability to culture from an infection is poor, specifically referencing 4 articles in which the accuracy rate ranged from $46 \%$ to 91\% (the $91 \%$ accuracy rate was from the Chew and Kline article discussed above). ${ }^{24}$

Meticulous evaluation of the statistics pertaining to the success rate of percutaneous biopsy in identifying vertebral osteomyelitis from large, general studies of vertebral lesions indicates that the success rates are lower. Early in 1998 Kornblum et al. evaluated CT-guided biopsy of the spine in 103 patients, in whom 90 adequate samples were obtained; 67 of the 90 samples were diagnostic; and 18 infections were identified based on cytological and/or pathological results, with 10 of 17 cases (58.8\%), yielding positive bacterial cultures. However, there appears to be overlap in the data and 19 cases of "normal tissue" are mentioned without any suggestion of false negatives. ${ }^{18}$ Enoch et al. reviewed 98 CT-guided procedures in 103 patients; only 9 of 25 samples (36\%) were positive for cultures. ${ }^{11}$ In a 2013 retrospective study of image-guided biopsy, Kim et al. reported a $97.1 \%$ overall success rate. They later claimed a $78.8 \%$ success rate $(134 / 170)$ in the diagnosis of infection; however, this statistic is based on the diagnosis of infection by histology or clinical outcome. In only 51/134 cases were causative organisms identified $(38.1 \%){ }^{17}$

There have been more and more studies recently focusing solely on biopsy for osteomyelitis and spondylodiscitis. As early as 1992, Fouquet et al. reviewed data obtained in 25 patients with discitis after lumbar surgery; of these, only in 9 cases were microorganisms identified $(36 \%) .{ }^{12}$ Hadjipavlou et al. in 2003 examined osteometabolic disorders in 68 patients, in whom a total of 71 biopsy samples were obtained and in whom a microbial diagnosis was established in $30(44 \%) .{ }^{15}$ Colmenero and associates $^{7,8}$ evaluated specifically for tuberculous osteomyelitis; in $2004,{ }^{7}$ they compared open to percutaneous biopsies and found $61.7 \%$ and $48 \%$ of samples to be positive, respectively. ${ }^{7}$ Yang and coworkers compared endoscopic and percutaneous techniques for biopsy and reported a positive result in $90 \%$ (18/20 patients) using endoscopic biopsy and in $47 \%$ (15/32 patients) using percutaneous biopsy. ${ }^{26}$ Ballah et al. recently examined osteomyelitis in children and found 12 of the 26 cases to be positive $(46 \%){ }^{2}$ The largest and most comprehensive was a report by Sehn and Gilula who indicated that 63 of 113 cases were positive when samples were examined histologically $(55.7 \%)$ and only 28 of the 92 cases positive when samples were examined microbiologically (30.4\%). ${ }^{22}$

Our current large study confirms the low yield from image-guided percutaneous biopsy when there is a high clinical suspicion of vertebral osteomyelitis; we observed positive histological results in only $38.2 \%(26 / 84)$ and positive microbiological results in $19.0 \%$ (16/84). In addition, of the 16 cases positive for microorganisms from biopsy, 8 cases, or $50 \%$, were also positive for blood cul- 
tures and urine cultures. Therefore, percutaneous biopsy for osteomyelitis helped to identify unknown microbes only in $8(9.5 \%)$ of 84 total cases. It is important to take note of Patzakis and colleagues' 1991 analysis of 61 patients with suspected osteomyelitis, in which 11 of 13 microbes $(85 \%)$ isolated from blood cultures corresponded to biopsy specimens, ${ }^{20}$ confirming a high correlation of systemic cultures for vertebral osteomyelitis.

Initially it was felt that biopsy should be performed only when a microoganism other than Staphylococcus was suspected or in situations in which there did not appear to be clinical evidence of improvement or normalization of laboratory findings after high-dose treatment. ${ }^{14}$ Authors such as Haaker et al. in 1997 recommended the immediate commencement of empirical antibiotic treatment when clinicians will later be able to narrow antibiotic coverage to the specific microbe discovered.14 However, it has now become routinely recommended that antimicrobial treatment not be started until after a biopsy sample is obtained except in cases of sepsis or neutropenia. ${ }^{5,9,13}$ Rankine and colleagues in 2004 , Grados et al. in 2006, and de Lucas and associates in 2008 have shown significant drops in their percentages of microbiology identification after antibiotics have commenced. Rankine et al. grew cultures from samples obtained in $50 \%$ of their patients $(6 / 12)$ prior to the use of antibiotics and in only $25 \%(2 / 8)$ after antibiotic initiation; Grados, in $80 \%$ of those not treated with antibiotics but only in $48 \%$ of those treated with antibiotics; and de Lucas, in $60 \%$ before and only $23 \%$ after antibiotic treatment. . $^{10,13,21}$

However, our comparison of the expected microbiology results from those patients who did not receive antibiotics prior to the percutaneous biopsy (9/44 or 20.5\%) with the observed results from patients who received antibiotics prophylactically (7/40 or $17.5 \%$ ) indicated that the results were similar for both groups and that the difference was not statistically significant $(p=0.4748)$. In other words, the proportion of positive cultures to those biopsied was low for both patients receiving and not receiving antibiotics prior to the biopsy, a finding in contrast to what has been previously reported. It is important to note that our study only obtained 16 positive microbiology results from the percutaneous biopsy; an analysis of a larger patient pool with a greater number of positive microbiology may demonstrate statistical significance and support the current guidelines to hold off prophylactic antibiotic treatment while awaiting culture results.

\section{Conclusions}

In summary, our department's extremely low yield of microorganisms recovered (16/84 or $19.0 \%$ ) from CTguided percutaneous biopsy confirms the findings seen in the study performed by Sehn and Gilula in 2012 in which positive microbiology was demonstrated in only 28 of 92 cases (30.4\%). ${ }^{22}$ Additionally, the data from the present study indicate that in only a smaller subset of patients (8/84 or $9.5 \%$ ) did the biopsy results provide clinicians with additional information as to specific microorganisms present and how treatment might need to be adjusted in light of these pathogens.
Image-guided percutaneous biopsy for suspected vertebral osteomyelitis is less likely to impact patient management than previously thought. Our data also suggest that prebiopsy antibiotic therapy does not significantly affect the pathogen recovery rate. A prospective trial is necessary to confirm these findings and determine whether any modifications to the current guidelines ought to be made. Until then, clinicians managing patients with suspected osteomyelitis should consider the results from this study when determining which patients should undergo image-guided biopsy. Patients in whom there are no clinical or imaging findings suspicious for atypical pathogens may be better managed with a course of empirical antibiotic therapy and without undergoing percutaneous intervention.

\section{Disclosure}

The authors report no conflict of interest concerning the materials or methods used in this study or the findings specified in this paper.

Author contributions to the study and manuscript preparation include the following. Conception and design: Young. Acquisition of data: Garg, Togaru. Analysis and interpretation of data: Garg. Drafting the article: Garg. Critically revising the article: Garg, Kosmas, Young. Reviewed submitted version of manuscript: Garg, Kosmas, Young, Robbin. Approved the final version of the manuscript on behalf of all authors: Garg. Statistical analysis: Garg. Study supervision: Robbin.

\section{References}

1. Ball RP: Needle (aspiration) biopsy. J Tenn Med Assoc 27: 203-206, 1934

2. Ballah D, Nijs E, Keller MS, Zhu X, Krishnamurthy G, Cahill AM: Percutaneous CT-guided vertebral bone biopsy in children. Pediatr Radiol 43:582-588, 2013

3. Carey TS, Evans AT, Hadler NM, Lieberman G, Kalsbeek WD, Jackman AM, et al: Acute severe low back pain. A population-based study of prevalence and care-seeking. Spine (Phila Pa 1976) 21:339-344, 1996

4. Carragee EJ: Pyogenic vertebral osteomyelitis. J Bone Joint Surg Am 79:874-880, 1997

5. Cheung WY, Luk KD: Pyogenic spondylitis. Int Orthop 36:397-404, 2012

6. Chew FS, Kline MJ: Diagnostic yield of CT-guided percutaneous aspiration procedures in suspected spontaneous infectious diskitis. Radiology 218:211-214, 2001

7. Colmenero JD, Jiménez-Mejías ME, Reguera JM, PalominoNicás J, Ruiz-Mesa JD, Márquez-Rivas J, et al: Tuberculous vertebral osteomyelitis in the new millennium: still a diagnostic and therapeutic challenge. Eur J Clin Microbiol Infect Dis 23:477-483, 2004

8. Colmenero JD, Ruiz-Mesa JD, Sanjuan-Jimenez R, Sobrino B, Morata P: Establishing the diagnosis of tuberculous vertebral osteomyelitis. Eur Spine J 22 (Suppl 4):579-586, 2013

9. Cottle L, Riordan T: Infectious spondylodiscitis. J Infect 56: 401-412, 2008

10. de Lucas EM, González Mandly A, Gutiérrez A, Pellón R, Martín-Cuesta L, Izquierdo J, et al: CT-guided fine-needle aspiration in vertebral osteomyelitis: true usefulness of a common practice. Clin Rheumatol 28:315-320, 2009

11. Enoch DA, Cargill JS, Laing R, Herbert S, Corrah TW, Brown NM: Value of CT-guided biopsy in the diagnosis of septic discitis. J Clin Pathol 61:750-753, 2008

12. Fouquet B, Goupille P, Jattiot F, Cotty P, Lapierre F, Valat JP, et al: Discitis after lumbar disc surgery: features of "aseptic", and "septic" forms. Spine (Phila Pa 1976) 17:356-358, 1992 


\section{Garg et al.}

13. Grados F, Lescure FX, Senneville E, Flipo RM, Schmit JL, Fardellone P: Suggestions for managing pyogenic (non-tuberculous) discitis in adults. Joint Bone Spine 74:133-139, 2007

14. Haaker RG, Senkal M, Kielich T, Krämer J: Percutaneous lumbar discectomy in the treatment of lumbar discitis. Eur Spine J 6:98-101, 1997

15. Hadjipavlou AG, Katonis PG, Tzermiadianos MN, Tsoukas GM, Sapkas G: Principles of management of osteometabolic disorders affecting the aging spine. Eur Spine J 12 (Suppl 2): S113-S131, 2003

16. Hendrickx L, Van Wijngaerden E, Samson I, Peetermans WE: Candidal vertebral osteomyelitis: report of 6 patients, and a review. Clin Infect Dis 32:527-533, 2001

17. Kim BJ, Lee JW, Kim SJ, Lee GY, Kang HS: Diagnostic yield of fluoroscopy-guided biopsy for infectious spondylitis. AJNR Am J Neuroradiol 34:233-238, 2013

18. Kornblum MB, Wesolowski DP, Fischgrund JS, Herkowitz HN: Computed tomography-guided biopsy of the spine. A review of 103 patients. Spine (Phila Pa 1976) 23:81-85, 1998

19. Matowe L, Gilbert FJ: How to synthesize evidence for imaging guidelines. Clin Radiol 59:63-68, 2004

20. Patzakis MJ, Rao S, Wilkins J, Moore TM, Harvey PJ: Analysis of 61 cases of vertebral osteomyelitis. Clin Orthop Relat Res (264): 178-183, 1991

21. Rankine JJ, Barron DA, Robinson P, Millner PA, Dickson RA:
Therapeutic impact of percutaneous spinal biopsy in spinal infection. Postgrad Med J 80:607-609, 2004

22. Sehn JK, Gilula LA: Percutaneous needle biopsy in diagnosis and identification of causative organisms in cases of suspected vertebral osteomyelitis. Eur J Radiol 81:940-946, 2012

23. Tali ET: Spinal infections. Eur J Radiol 50:120-133, 2004

24. Tehranzadeh, J, Tao C, Browning CA: Percutaneous needle biopsy of the spine. Acta Radiol 48:860-868, 2007

25. Wirtz DC, Genius I, Wildberger JE, Adam G, Zilkens KW, Niethard FU: Diagnostic and therapeutic management of lumbar and thoracic spondylodiscitis - an evaluation of 59 cases. Arch Orthop Trauma Surg 120:245-251, 2000

26. Yang SC, Fu TS, Chen LH, Chen WJ, Tu YK: Identifying pathogens of spondylodiscitis: percutaneous endoscopy or CT-guided biopsy. Clin Orthop Relat Res 466:3086-3092, 2008

Manuscript submitted April 14, 2014.

Accepted June 2, 2014.

Please include this information when citing this paper: DOI: 10.3171/2014.6.FOCUS14134.

Address correspondence to: Vasant Garg, M.D., Department of Radiology, University Hospital Case Medical Center, 11100 Euclid Ave., Cleveland, OH 44106. email: vasant.garg@uhhospitals.org. 